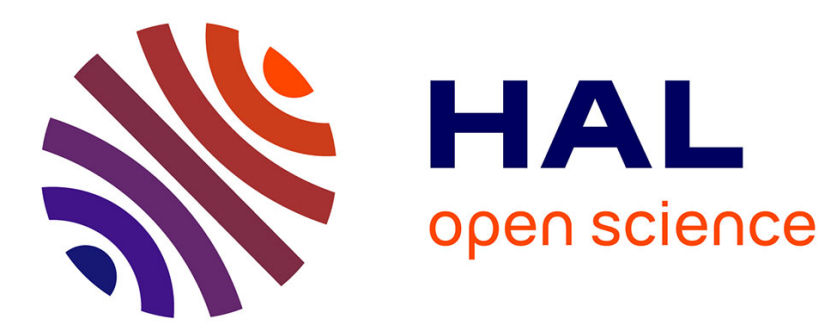

\title{
Application of the Thermodynamic Extremal Principle to Modeling of Thermodynamic Processes in Material Sciences
}

\author{
Jiri Svoboda, Ilja Turek, Franz Dieter Fischer
}

\section{- To cite this version:}

Jiri Svoboda, Ilja Turek, Franz Dieter Fischer. Application of the Thermodynamic Extremal Principle to Modeling of Thermodynamic Processes in Material Sciences. Philosophical Magazine, 2005, 85 (31), pp.3699-3707. 10.1080/14786430500267760 . hal-00513586

\section{HAL Id: hal-00513586 \\ https://hal.science/hal-00513586}

Submitted on 1 Sep 2010

HAL is a multi-disciplinary open access archive for the deposit and dissemination of scientific research documents, whether they are published or not. The documents may come from teaching and research institutions in France or abroad, or from public or private research centers.
L'archive ouverte pluridisciplinaire HAL, est destinée au dépôt et à la diffusion de documents scientifiques de niveau recherche, publiés ou non, émanant des établissements d'enseignement et de recherche français ou étrangers, des laboratoires publics ou privés. 


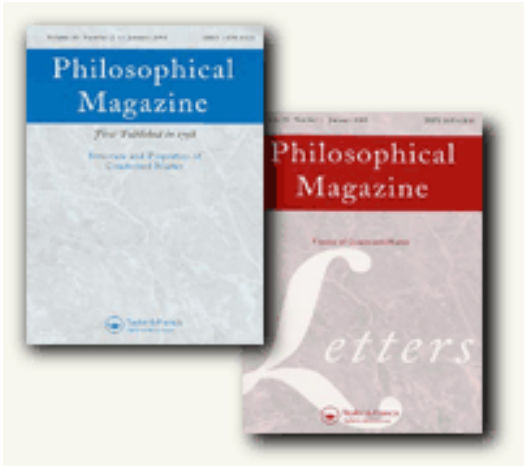

\section{Application of the Thermodynamic Extremal Principle to Modeling of Thermodynamic Processes in Material Sciences}

\begin{tabular}{|r|l|}
\hline Journal: & Philosophical Magazine \& Philosophical Magazine Letters \\
\hline Manuscript ID: & TPHM-05-May-0138 \\
\hline Journal Selection: & Philosophical Magazine \\
\hline Author: & 03-May-2005 \\
\hline Complete List of Authors: & $\begin{array}{l}\text { Svoboda, Jiri; Institute of Physics of Materials, Academy of Sciences } \\
\text { of the Czech Republic } \\
\text { Turek, Ilja; Institute of Physics of Materials, Academy of Sciences of } \\
\text { the Czech Republic } \\
\text { Fischer, Franz Dieter; Erich Schmid Institute for Materials Science, } \\
\text { Austrian Academy of Sciences }\end{array}$ \\
\hline Keywords: & thermodynamics, modelling, diffusion, phase transformations \\
\hline Keywords (user supplied): & $\begin{array}{l}\text { thermodynamic extremal principle, evolution equations, reciprocal } \\
\text { relations }\end{array}$ \\
\hline &
\end{tabular}

\section{s) ScholarONE \\ Manuscript Central}


1

2

3

\section{Application of the Thermodynamic Extremal Principle to} Modeling of Thermodynamic Processes in Material Sciences

\section{Short Title: Thermodynamic Extremal Principle}

\section{$\mathrm{J}_{\sharp}$ SVOBODA $^{\left.1^{*}\right)}, \mathrm{I}_{\rightarrow}$ TUREK $^{1}$, and F.D. $\mathrm{PISCHER}_{\exists}^{2}$}

${ }^{1}$ Institute of Physics of Materials, Academy of Sciences of the Czech Republic, Žižkova 22, CZ-61662 Brno, Czech Republic, Tel.: 0042 05/32 2904 07, Fax: 0042 05/41 2186 57, email: svobj@ipm.cz

${ }^{2}$ Institute of Mechanics, Montanuniversität Leoben, Franz-Josef-Strasse 18, A-8700 Leoben, Austria and Erich Schmid Institute for Materials Science, Austrian Academy of Sciences, Jahnstrasse 12, A-8700 Leoben, Austria.

\section{Abstract}

An extremal principle is formulated for thermodynamic systems near equilibrium subjected to various external conditions. It is shown that the principle describes unambiguously the kinetics of the thermodynamic system and replaces classical phenomenological equations. The global formulation of the principle makes it possible I) to introduce discrete characteristic parameters for the proper description of the state and evolution of the system, II) to take into account constraints amongst the characteristic parameters of the local or global character and III) to derive the evolution equations for the characteristic parameters. These facts turn the principle into an effective tool for the treatment of non-equilibrium thermodynamic systems.

Keywords: Non-equilibrium thermodynamics, phenomenological equations, thermodynamic extremal principle, evolution equations, reciprocal relations

\footnotetext{
*) Corresponding author
}

\section{Formatted}

Deleted: under

Deleted: substitutes the

Deleted: some

Deleted: a

Deleted: b

Deleted: c 


\section{Introduction}

The classical treatment of linear thermodynamics of irreversible processes represents a considerably complicated system of linear relations amongst local thermodynamic quantities [1-3]. It is suitable e.g. for modeling of material processing and properties at elevated temperatures. However, it is necessary to admit that linear thermodynamics of irreversible processes is not a general tool for the treatment of the time evolution of arbitrary systems, for a detailed discussion see [4].

The systems treated in material science are usually rather complex. $\llbracket n$ most cases however, only a limited number of characteristic parameters (CPs) can be used for the description of the state and evolution of the system and only a limited number of CPs are of interest implying a certain degree of idealization of the system. The number of CPs depends on the required accuracy of the description and on the complexity of the system. Moreover, constraints of local or global character amongst the CPs must often be taken into account. The goal is the determination of the evolution equations for the $\mathrm{CPs}_{\mathrm{s}}$

The classical way of determining the evolution of a system is represented by the solution of phenomenological equations complemented by conservation laws and proper boundary and contact as well as initial conditions. By setting some assumptions on the system geometry a certain degree of idealization of the system can be achieved, so that the phenomenological equations can be solved providing the time evolution of the CPs. However, in many practical cases the amount of necessary idealization may completely be lacking of the physical substance of the problem. Obviously, the solution of the standard phenomenological equations need not to be the most efficient way. This problem could be overcome by the application of the Thermodynamic Extremal Principle (TEP), which represents a tool for deriving evolution equations for the CPs in a direct way but is very rarely exploited. The present paper is aimed to show a concept how the TEP can be applied.

The story of TEP starts with the variational formulation of a thermodynamic problem by Onsager [5] in 1931, who showed that the equations for heat conduction in an anisotropic system can be derived from the requirement of the maximum of a functional having a close relation to the total entropy production in the system. During the following 60 years the field of extremal principles in thermodynamics has spread in several directions. The Lagrangian [6, 7] and Hamiltonian [8,9] structures of the principle as well as the method of path integrals [10] have been developed. Furthermore, extremal principles have been formulated for steady-

Deleted: On the one hand i
Deleted: It
Deleted: On the other hand

Deleted: However, i

Deleted: and only a limited number of CPs

Deleted: our

Deleted: to us. This limitation

Deleted: ies

Deleted: a lower or higher

Deleted: is usually required

Deleted: kinetics

Deleted:
Deleted: and
Deleted: and
Deleted:
Deleted: The
Deleted: can be obtained from the
solution of the phenomenological
equations.
Deleted: be


state and transient non-linear systems or systems far from equilibrium [7, 11, 12]. The important role of entropy production with respect to the proper formulation of constitutive laws in a wide field of applications can be recognized in [13-15]. It was also demonstrated, that the principle can be generalized to arbitrary processes obeying the laws of linear nonequilibrium thermodynamics [16-18].

During the last 15 years the TEP has been applied to the development of models in materials science [19-28]. The authors have demonstrated that the TEP seems to be a handy tool for the solution of practical problems of thermodynamics of irreversible processes, which are often unsolvable or solvable only with great complications in the conventional way. However, apparently the TEP and its application to modeling have still not penetrated sufficiently into the material sciences community. Therefore, the aim of this paper is to demonstrate a generally applicable concept of the TEP which can be a guide for modeling of a large class of problems in material sciences.

\section{Formulation of the Thermodynamic Extremal Principle (TEP)}

Let us consider a body and define in its interior a system occupying the domain $V$ with the surface $\partial V$ and the surface normal $\underline{n}$ in its actual configuration at time $t$. The Cartesian coordinates $x_{1}, x_{2}, x_{3}$ are represented by a vector $\underline{x}$. Scalar quantities like the temperature $\theta$ are functions of $\underline{x}$ and $t, \theta=\theta(\underline{x}, t)$. Vectors are denoted by e.g. $\underline{a}$, second order tensors are denoted by e.g. $R$. We use the Einstein summation convention for double indices meaning $a_{i} b_{i}=\sum_{i=0}^{n} a_{i} b_{i}$. The sum $\underline{b}_{k}^{T} \cdot \underline{j}_{k}$ means the sum of inner products of the vectors $\underline{b}_{k}$ and $\underline{j}_{k}$ over $k$; the superscript " $T$ " refers to a transposed vector, tensor or matrix. The product $a^{T} \cdot \underline{R} \cdot \underline{a}$ is a quadratic form in $a_{1}, a_{2}, a_{3}$, meaning the inner product of the vector $a$ with the vector $\underline{\underline{R}} \cdot \underline{a}$ being the matrix product of $\underline{R}$ and $\underline{a}$.

Let the thermodynamic system be near the thermodynamic equilibrium. The evolution of the system is caused by irreversible processes in the system. These processes are represented by fluxes, by the motion of interfaces in polycrystalline and/or multiphase systems and finally the rates of eigenstrain components. For simplification we consider only the fluxes as kinetic variables in the system. Let $j_{k}=j_{k}(\underline{x}, t), k=0,1,2, \ldots, n$, be the fluxes
Deleted: Onsager's original formulation of

Deleted: s
Deleted: tensor

Deleted: The matrix $R$ is selected as a symmetric one with only real eigenvalues.

Deleted: and/or

Deleted: ; the motion of the interfaces can be treated analogously 
From the requirement that the entropy production $\underline{\mathrm{P}}$ in any part of the system $\underline{\text { must }}$ be zero in the state of thermodynamic equilibrium, $\underline{j}_{k} \equiv \underline{0}$, it follows that $A \equiv 0$. Since for systems not in thermodynamic equilibrium the total entropy production $P$ must be positive for arbitrary fluxes (Second Law of Thermodynamics), also the second term in the expansion (1) must not appear, $\underline{B}_{k} \equiv \underline{0}$, and the third term must be a positive definite quadratic form of the components of the fluxes $\underline{j}_{k}$. For the state near the thermodynamic equilibrium small values of fluxes $\underline{j}_{k}$ can be expected and, therefore, the higher terms in eqn. (1) are negligible. Then $P$ is given by the truncated Taylor series after three terms as

$$
P=\int_{V} \underline{j}_{k}^{T} \cdot \underline{\underline{R}}_{\ell} \cdot \underline{j}_{\ell} d V \equiv 2 \phi\left(\underline{j}_{k}, \underline{j}_{\ell}\right) \geq 0
$$

\section{Let $j_{k i}$ be the $i$-th component of the flux $\underline{j}_{k} 2 j_{\ell j}$ be the $j$-th component of the flux $\underline{j}_{\ell}$ and} $R_{k \ell i j}$ be the $i j$ element of the matrix ${ }_{k} \underset{=}{R}$. Then $R_{k \ell i j}=\frac{1}{2} \frac{\partial^{2} P}{\partial j_{k i} \partial j_{\ell j}}=\frac{1}{2} \frac{\partial^{2} P}{\partial j_{\ell j} \partial j_{k i}}=R_{\ell k j i}$, and the symmetry relation in the form ${ }_{k} \underline{\underline{R}}={ }_{\ell} \underline{\underline{R}}_{k}^{T}$ must be fulfilled. ${ }_{k} \underline{\underline{R}}$ - describes the local material properties, The quantity $P$, equation (2) is in its substance the mathematical expression of the Second Law of Thermodynamics for systems near the thermodynamic equilibrium being a positive definite quadratic form in the fluxes.

The rate of the total entropy, $\dot{S}$, of an open system is given by

$$
\dot{S}=P+\dot{S}_{\text {ext }},
$$

\begin{tabular}{l} 
Deleted: \\
Deleted: \\
\hline Deleted:
\end{tabular}

Deleted: out of

Deleted: and must be a positive definite matrix to assure the inequality (2) Deleted: . No symmetry properties of the tensor $\underset{k}{R} \ell$ are expected a priori. E Deleted: second Deleted: law Deleted: thermodynamics Deleted: the 
where $\dot{S}_{\text {ext }}$ is the total flow of the entropy into the system. The rate of the total entropy, $\dot{S}$,

can then be expressed according to [2] as

$$
\dot{S}=\int_{V} \frac{1}{\theta} \mu_{k} \operatorname{div}\left(\underline{j}_{k}\right) d V
$$

where $\mu_{k}(\underline{x}, t), k=1,2, \ldots, n$, are the chemical potentials of the components $k$ and $\mu_{0}=-1$.

The total flow of the entropy into the system follows according to [2] as

$$
\dot{S}_{\text {ext }}=\int_{\partial V} \frac{1}{\theta} \mu_{k} \underline{j}_{k}^{T} \cdot \underline{n} d A
$$

The combination of eqns. (4) and (5) yields, by the application of the Gauss theorem,

$$
P=\dot{S}-\dot{S}_{e x t}=-\int_{V} \underline{j}_{k}^{T} \cdot \operatorname{grad}\left(\frac{\mu_{k}}{\theta}\right) d V \equiv \psi\left(\underline{j}_{k}\right)
$$

\section{The quantity $P$, equation (6), stemming from balancing the rate of entropy in an open system,} is obviously a linear form in the fluxes.

A comparison of eqns. (2) and (6) furnishes the necessary equivalence condition

$$
2 \phi\left(\underline{j}_{k}, \underline{j}_{\ell}\right)=\psi\left(\underline{j}_{k}\right) .
$$

Both functionals $s_{2} 2 \phi\left(\underline{j}_{k}, \underline{j}_{\ell}\right)$ and $\psi\left(j_{k}\right)_{2}$ represent the total entropy production in the system. They have, however, a different physical meaning. As outlined above $2 \phi\left(\underline{j}_{k}, \underline{j}_{\ell}\right)$ is a positive definite quadratic form of the components of fluxes and reflects the material properties of the system. As a simple example it corresponds to the heat produced in a resistor due to an electric current. On the other hand $\psi\left(j_{k}\right)$ is a linear form of the components of fluxes and reflects the driving forces in the system. In the a.m. simple example it corresponds to the energy released by the motion of electrons in an electric field caused by the voltage applied on the resistor.

Now the Thermodynamic Extremal Principle (TEP), is formulated as follows: 
From all admissible fluxes $\underline{j}_{k}$, constrained by some conservation and boundary/contact conditions, those are selected in an irreversible process in a thermodynamic system near the equilibrium, which maximize the total entropy production in the system, expressed either by $2 \phi\left(j_{k}, j_{\ell}\right)$ or by $\psi\left(j_{k}\right)$ and subjected to the equivalence condition (7).

The last condition (7) involves via the driving force the "physical character" of the problem.

\section{Derivation of Phenomenological Equations}

The requirement of an extremum of the functional $\psi$ with respect to the fluxes $\underline{j}_{k}$, constrained by condition (7), leads to the variation

$$
\delta(\psi+\alpha(2 \phi-\psi))=0
$$

with $\alpha$ being a Lagrange multiplier. Using relations (2) and (6) the variation (8) leads to the Euler/Lagrange equations

$$
-\operatorname{grad}\left(\frac{\mu_{k}}{\theta}\right)+\alpha\left(2{ }_{k} \underline{\underline{R}} \cdot \underline{j}_{\ell}+\operatorname{grad}\left(\frac{\mu_{k}}{\theta}\right)\right)=\underline{0}, k=0,1, \ldots, n
$$

Multiplication of equations (9) by $\underline{j}_{k}$ from the left side, their summation with respect to k, integration over the volume $V$ of the system and comparison with eqn. (7) yield the value of $\alpha=-1$. Equations (9) can then be rewritten as

$$
{ }_{k} \underline{R}_{\ell} \cdot \underline{j}_{\ell}=-\operatorname{grad}\left(\frac{\mu_{k}}{\theta}\right), k=0,1, \ldots, n
$$

Relations (10), representing 3 linear equations for each $k$ and altogether $3(n+1)$ linear equations for the $3(n+1)$ unknown components of the fluxes $\underline{j}_{0}, \ldots . \underline{j}_{n}$, can formally be assembled into a matrix equation $\overline{\bar{R}} \cdot \bar{j}=\bar{f}$ with a $3(n+1) \times 3(n+1)$ matrix $\overline{\bar{R}}$ consisting of the submatrices ${ }_{k} \underline{R}_{\ell^{2}}$ the vector $\bar{j}$ consisting of the subvectors $\underline{j}_{k}$ and the vector $\bar{f}$

\section{Field Code Changed}

Deleted: ), $2 \phi \equiv \psi$ which

introduces the "physics behind" in the variational problem

Deleted: The irreversible processes in a thermodynamic system near the

equilibrium, given by fluxes $j_{k}$, lead to a maximum total entropy production in the system given either by functional $2 \phi\left(j_{k}, j_{\ell}\right)$ (eqn. (2)) or $\psi\left(j_{k}\right)$ (eqn. (6)), constrained by the equivalence condition (7) and, if any, by further conditions stemming from the nature of the problem. II

\footnotetext{
Deleted: The matrix $\underset{k}{\underline{R}} \ell$ is given by $\mathscr{I}$ II $\underline{\underline{R}}_{\ell}=\frac{1}{2}\left({ }_{k} \underline{\underline{R}}_{\ell}+{ }_{\ell} \underline{\underline{R}}_{k}^{T}\right)$ with ${ }_{k} \underline{\underline{R}}_{\ell}={ }_{\ell} \underline{\underline{R}}_{k}^{T} \ldots(11) \mathrm{d}$ II and is equal to ${ }_{\mathrm{k}} \underline{\underline{\mathrm{R}} \ell}$ being a positive definite matrix.II

Deleted: every

Deleted: $\underline{R}_{\ell}$,
} 
consisting of the subvectors $-\operatorname{grad}\left(\frac{\mu_{k}}{\theta}\right)$. The matrix $\overline{\bar{R}}$ is then symmetric due to ${ }_{k} \underline{\underline{R}}_{\ell}={ }_{\ell} \underline{\underline{R}}_{k}^{T}$ The integrand of eqn. (2) can now be rewritten as $\bar{j}^{T} \cdot \overline{\bar{R}} \cdot \bar{j}$. Since $P$ must be positive for any volume $V$, this fact leads directly to the positive definiteness of $\overline{\bar{R}}$ allowing an inversion of the matrix relation $\overline{\bar{R}} \cdot \bar{j}=\bar{f}$ to $\bar{j}=\overline{\bar{L}} \cdot \bar{f}$. The matrix $\overline{\bar{L}}$ is also positive definite and symmetric and can be disassembled into the submatrices ${ }_{k} \underline{\underline{L}} \underline{\underline{\ell}}_{\ell}$. Then the kinetic equations can be written in the form

$$
\underline{j}_{k}=-{ }_{k} \underline{\underline{L}}_{\ell} \cdot \operatorname{grad}\left(\frac{\mu_{\ell}}{\theta}\right)
$$

which are the linear, ${ }_{2}$ phenomenological equations, see e.g. [1-3].

Since the properties of the matrices $\overline{\bar{R}}$ and $\overline{\bar{L}}$ are the same, the properties of ${ }_{k} \underline{\underline{R}}$ can be identically addressed to ${ }_{k} \underline{\underline{L}}_{\ell}$. Thus the symmetry relation for ${ }_{k} \underline{\underline{L}}_{\ell}$ reads

$$
{ }_{k} \underline{\underline{L}}_{\ell}={ }_{\ell} \underline{\underline{L}}_{k}^{T} .
$$

For the isotropic cases ${ }_{k} \underline{\underline{L} \ell}$ takes the form of $L_{k \ell} \underline{I}, \underline{\underline{I}}$ unity tensor. The coefficients $L_{k \ell}$ have the symmetry properties $L_{k \ell}=L_{\ell k}$ and can be addressed as the well-known Onsager's reciprocal relations. In general cross-effects are met by eqn. (12) in anisotropic materials ${ }_{-1}$ It should be emphasized that the symmetry relations are a direct consequence of the application of TEP and they were not a priori assumed as strongly criticized e.g. by Truesdell [29], Lecture 7. They stem from the assumption that the entropy production is a positive definite quadratic form of the kinetic variables for states near the thermodynamic equilibrium.

Insertion of $\alpha=-1$ into eqn. (8) yields $(-2 \phi+2 \psi)$ representing the functional of the entropy production, $P$, constrained by eqn. (7) to be extremized. The functional can be rewritten as $\int_{V}\left(-\bar{j}^{T} \cdot \overline{\bar{R}} \cdot \bar{j}+2 \bar{j}^{T} \cdot \bar{f}\right) d V$. The second partial derivatives of the integrand with respect to $\bar{j}$ yield the Hessian matrix $-2 \overline{\bar{R}}$. Since $\overline{\bar{R}}$ is a positive definite matrix, the negative definite Hessian matrix $-2 \overline{\bar{R}}$ ensures a maximum of the functional $(-2 \phi+2 \psi)$.
Deleted: definition (11).

Deleted: evolution

Deleted: 13

Deleted: Note that for $k \neq \ell$ the
matrices $\tilde{L}_{k=\ell}$ are not symmetric.
Deleted: $L_{k \ell}$ are
Deleted: with
Deleted: 13
Deleted: and by $L_{k \ell}=L_{\ell k}$ in
isotropic materials.
Deleted: is necessary to stress,
Deleted: $\mathrm{m}$
Deleted: in the case




\section{Thermodynamic Potentials}

The formulation of the TEP presented in Section 2 is designed for the most general open thermodynamic systems. In practice many thermodynamic systems can be considered as closed systems. In the following it is shown that for closed systems the rate of a thermodynamic potential takes over the role of the total entropy production.

The simplest system cannot exchange the entropy with its surroundings enforcing $\dot{S}_{e x t}=0$, i.e. an insulated closed system with a fixed volume $V$. Then according to eqn. (3) the total entropy production in the system $P$ equals the rate of the total entropy of the system $\dot{S}$

$P=\dot{S}$

For such a system the TEP can be formulated in terms of $\dot{S}$ replacing $P$. As a direct consequence of the TEP $\dot{S}>0$ for states out of equilibrium ${ }_{2}$ and $S$ achieves its maximum for the equilibrium state of the system.

Systems at a constant temperature $\theta$ are often a better approximation of the reality than insulated ones. Such a condition can be ensured by a constant environment temperature and by a sufficiently high heat conductivity in the system. Assuming a constant volume $V$ of the system, it follows according to the first law of thermodynamics that

$$
\dot{E}=\theta \dot{S}_{\text {ext }}
$$

$E$ is the total internal energy of the system. According to eqn. (6)

$$
P=\frac{\theta \dot{S}-\dot{E}}{\theta}=-\frac{\dot{F}}{\theta}
$$

where $F$ is the total free energy of the system.

In the case of a constant temperature $\theta$ and a constant external pressure $p$, the first law of thermodynamics reads
Deleted: most

Deleted: 14

Deleted: then according to the first law of thermodynamic

Deleted: 15

Deleted: where

Deleted: 16 


\section{Formulation of the Thermodynamic Extremal Principle in Terms of}

\section{Characteristic Parameters (CPs)}

Let us consider a thermodynamic system under certain external conditions to which a thermodynamic potential $X$ corresponds $(X \equiv S$ for $E=$ const and $V=$ const,$X \equiv-F / \theta$ for $\theta=$ const and $V=$ const,$X \equiv-G / \theta$ for $\theta=$ const and $p=$ const $)$. The replacement of the total entropy production $P$ by the rate of the thermodynamic potential, $\dot{X}$, simplifies the treatment of closed systems. We assume that the thermodynamic potential $X$, as a global state parameter of the system, can be expressed as a function $X=X\left(q_{1}, q_{2}, \ldots, q_{N}\right)$ of the set of the CPs denoted by $q_{i}(i=1, \ldots . N)$. The time evolution of the system is caused by fluxes $\underline{j}_{\ell}$, $\ell=0, \ldots . . n$, introduced in Section 2, and by the motion of the migrating interfaces. The time evolution results in the change of CPs described by the rates of the CPs denoted by $\dot{q}_{i}$ $(i=1, \ldots . N)$, which were denominated as "kinetic variables" in section 2.

We expect that using mass conservation laws, proper simplifications and assumptions on the geometry of the system, the linear relations (the Einstein summation convention for double indices is used again)

$$
\underline{j}_{k}=f_{i k}\left(q_{1}, q_{2}, \ldots . q_{N}\right) \dot{q}_{i}, \quad k=0, \ldots ., n
$$


can be found. The total entropy production due to dissipation in the isotropic system is given

Deleted: $v$ is the local interface velocity. by a general relation $[2,27]$

$$
2 \phi=\int_{V} \frac{R_{g} \underline{j}_{k}^{2}}{c_{k} D_{k}} d V+\int_{V} \frac{\underline{j}_{0}^{2}}{\theta^{2} \lambda} d V+\int_{\text {Interfaces }} \frac{v^{2}}{\theta M} d A
$$

$R_{g}$ is the gas constant, $c_{k}$ the concentration of component $k, D_{k}$ the tracer diffusion coefficient of component $k, M$ the interface mobility and $\lambda$ the heat conductivity. After inserting eqns. (18) and (19) into eqn. (20) and performing the integrals one obtains the functional $2 \phi$ in the form

$$
2 \phi=U_{i j}\left(q_{1}, q_{2}, \ldots . q_{N}\right) \dot{q}_{i} \dot{q}_{j}
$$

The matrix $U_{i j}$ is positive definite and takes into account the material properties and geometry of the system. The functional $\psi$ can be calculated due to eqns. (13), (15) and (17) as

$$
\psi=\dot{X}=\frac{\partial X}{\partial q_{i}} \dot{q}_{i} .
$$

Deleted: I
Deleted: 19
Deleted: 20
Deleted: 21
Deleted: 22

Deleted: and symmetric

Deleted: 18

Deleted: 23

We assume $m$ constraints amongst the rates of CPs having the form

$$
a_{i k} \dot{q}_{i}=0 ; \quad k=1,2, \ldots ., m .
$$

Then the necessary condition for an extremum of $\psi_{2}$ constrained by eqs. (7) and (23) $)_{2}$ leads to the equations

Deleted: the maximum Deleted: 24 Deleted: :

Deleted: 25 
The quantities $\alpha$ and $\beta_{k}$ are the Lagrange multipliers. Using eqs. (21) and (22) and performing the derivatives one obtains

$$
\frac{\partial X}{\partial q_{i}}+\alpha\left(2 U_{i j} \dot{q}_{j}-\frac{\partial X}{\partial q_{i}}\right)+2 \beta_{k} a_{i k}=0
$$

Multiplication of eqn. (25) by $\dot{q}_{i}$, summation over $i$ and the use of eqs. (21), (22) and (23) lead to

$$
\psi+\alpha(4 \phi-\psi)=0
$$

Comparison of eqn. (26) with eqn. (7) gives immediately $\alpha=-1$. Then the resulting set of linear equations for the system evolution follows as

$$
U_{i j} \dot{q}_{j}+a_{i k} \beta_{k}=\frac{\partial X}{\partial q_{i}}
$$

which can be solved together with eqns. (23) with respect to $\dot{q}_{i}(i=1,2, \ldots N)$ and $\beta_{k}(k=1,2, \ldots, m)$. The integration of $\dot{q}_{i}$ with respect to time provides the description of the system evolution by means of evolution of the chosen CPs $q_{i}(t)$.

\section{6._Comments on the Principle of the Least Dissipation of Energy and the Principle of the Minimum Entropy Production}

Onsager's Principle [5] is often called the Principle of the Least Dissipation of Energy. This denotation is, however, misleading, since according to Onsager's Principle the evolution of the system corresponds to the constrained maximum of dissipation. Onsager himself tried to show [5] that for steady states with prescribed fluxes on the system surface $\partial V$ his Principle becomes an assertion that the dissipation assumes a minimum. Onsager's treatment is, however, not correct. The steady states are characterized by $\operatorname{div}\left(j_{i}\right)=\underline{0}$ and thus $\dot{S} \equiv 0$ (see 
eqn. (4)). Onsager argued that for fixed values of $j_{k}^{T} \cdot \underline{n}$ on the whole system surface $\partial V$ also $\dot{S}_{\text {ext }}$ were fixed. This is, however, not the case as the quantity $\mu_{k} / \theta$ is not fixed on the system surface $\partial V$, see eqn. (5). Under this wrong assumption $\psi\left(j_{k}\right)$ is fixed and for $(-2 \phi+2 \psi)$ assuming to be a maximum, the quantity $P=2 \phi$ assumes a minimum (see the end of Section 3). Unfortunately, the same wrong treatment is also used as a key argument by Gyarmati (see page 111 in [18]).

The steady states are analyzed in detail by means of the Principle of the Minimum Entropy Production in [2]. It is shown that for fixed values of $\mu_{i}$ and $\theta$ on the whole system surface $\partial V$ and under the assumption of Onsager's "reciprocal relations" the steady states correspond to the minimum of the entropy production ${ }_{2}$ and these states are stable relative to perturbations. To the knowledge of the authors, a conclusive proof is still missing that the Principle of the Minimum Entropy Production is a direct consequence of Onsager's Principle.

\section{Conclusion}

Based on the application of TEP an alternative approach to the treatment of thermodynamic systems near the equilibrium is reported with the following results:

1. On the basis of purely thermodynamic considerations the Thermodynamic Extremal Principle (TEP) is formulated and can be considered as a generalized form of Onsager's principle [5] for the maximum dissipation rate in the case of heat conduction.

2. The classical phenomenological equations describing the system evolution on the local level and including Onsager's reciprocity relations are derived from the TEP. This is a direct consequence that the dissipation rate is approximated in the sense of a truncated Taylor series by a positive definite quadratic form in the thermodynamic fluxes.

3. Thermodynamic potentials are assigned to closed thermodynamic systems under various external conditions. The rates of the thermodynamic potentials take over the role of entropy production in the closed systems.

4. The TEP for closed systems is formulated for a discrete set of CPs allowing an optimal description of the state and evolution of the system on the discretized global level.

Deleted: Furthermore,
Deleted: argues
Deleted: is
Deleted:
Deleted: which

Deleted: A

Deleted: representing a generalized form of Onsager's principle [5] 


\section{ACKNOWLEDGEMENTS}

The authors are indebted to Materials Center Leoben and the Austrian Kplus programme for financial support within the strategic project MCL SP16. The work on the project was also partly supported by the Academy of Sciences of the Czech Republic under the Grant 1QS200410502. 


\section{REFERENCES}

[1] H.B. Callen, Thermodynamics, (Wiley, New York, 1960).

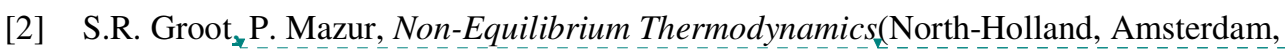
1962).

[3] I. Prigogine, Introduction to Thermodynamics of Irreversible Processes, (Interscience, New York, 1967), $3^{\text {rd }}$ edn.

[4] K. R. Rajagopal A. R. Srinivasa, Z. angew. Math. Phys. 55, 861 (2004).

[5] L. Onsager, Phys. Rev. 37, 405 (1931).

[6] R.J. Donnelly, R. Herman 2 I. Prigogine, Non-Equilibrium Thermodynamics, Variational Techniques and Stability (The University of Chicago Press, Chicago, 1967), $2^{\text {nd }} \mathrm{edn}$.

[7] R.E. Nettleton, J. Phys. A: Math. Gen. 19, L295 (1986).

[8] S. Sieniutycz, R.S. Berry, Phys Rev A 40, 348 (1989).

[9] E. Vázquez, J.A. del Rio _ M. López de Haro, Phys Rev $_{\sharp}$ E 55, 5033 (1997).

[10] R. Graham, Z. Physik B 26, 281 (1977).

[11] C. Garrod, J. Non-Equilib. Thermodyn. 9, 97 (1984).

[12] H. Grabert M.S. Green, Phys. Rev. A19, 1747 (1979).

[13] I. Müller T. Ruggeri, Extended Thermodynamics (Springer-Verlag, Berlin, 1993).

[14] D. Jou, J. Casas-Vázquez _ G. Lebon, Extended Irreversible Thermodynamics (SpringerVerlag, Berlin, Heidelberg, New York et.al. 1993).

[15] A. Kleidon ${ }_{\star}$ R.D. Lorenz, Non-Equilibrium Thermodynmics and the Production of Entropy (Springer-Verlag, Berlin, Heidelberg, New York et.al. 2005).

[16] H. Ziegler, Ing.-Arch 30, 410 (1961).

[17] H. Ziegler, in Progress in Solid Mechanics, Vol. 4; edited by I.N. Sneddon and R. Hill (North-Holland, Amsterdam, 1963), pp. 91-193.

[18] I. Gyarmati, Non-Equilibrium Thermodynamics. (Springer-Verlag, Berlin, Heidelberg, New York, et.al. 1970).

[19] J. Svoboda, I. Turek, V. Sklenička, Acta Metall. Mater. 38, 573 (1990).

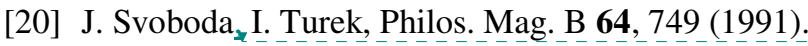

[21] A.C.F. Cocks, S.P.A. Gill, J.-Z. Pan, in Advances in Applied Mechanics; edited by E. vd. Giessen and Th.Y. Wu (Academic Press, San Diego et al., 1999), Vol. 36, pp. 81 162.

[22] S. P.A. Gill, M. G. Cornforth A. C.F. Cocks, Int. J. Plasticity 17, 669 (2001).

[23] J. Svoboda ${ }_{\varkappa}$. Lukáš, Acta Mater. 48, 2519 (2000).

[24] J. Svoboda, F. D. Fischer, P. Fratzl A. Kroupa, Acta Mater. 50, 1369 (2002).

Deleted: and
Deleted: and
Deleted: and
Deleted: .

\begin{tabular}{l} 
Deleted: ( \\
Deleted: and \\
Deleted: \\
Deleted: $3^{\text {rd }} \mathrm{edn}$. \\
\hline
\end{tabular}

Deleted: and

Deleted: a

Deleted: and

Deleted: $2^{\text {nd }} \mathrm{Ed}$

Deleted: and
Deleted: ical
Deleted: iew
Deleted: and
Deleted: ical
Deleted: iew
Deleted: and and
Deleted: and

Deleted: and
Deleted: and

Deleted: and

Deleted: and 
1

2

3

4

5

6

7

8

9

10

11

12

13

14

15

16

17

18

19

20

21

22

23

24

25

26

27

28

29

30

31

32

33

34

35

36

37

38

39

40

41

42

43

44

45

46

47

48

49

50

51

52

53

54

55

56

57

58

59

60
[25] F. D. Fischer, J. Svoboda, P. Fratzl, Phil, Mag. A 83, 1075 (2003).

[26] J. Svoboda, E. Gamsjäger, F. D. Fischer, P. Fratzl, Acta Mater. 52, 959 (2004).

[27] J. Svoboda, F. D. Fischer, P. Fratzl_E. Kozeschnik, Mater. Sci. Eng, A, 385, 166 (2004).

[28] Kozeschnik E., J. Svoboda, P. Fratzl _F. D. Fischer, Mater. Sci. Eng, A, 385, 157 (2004).

[29] C. Truesdell, Rational Thermodynamics (Springer-Verlag, New York Berlin Heidelberg Tokyo, 1984), $2^{\text {nd }}$ edt., pp. 365-395.

\begin{tabular}{l} 
Deleted: and \\
Deleted: os \\
Deleted: and \\
Deleted: and \\
Deleted: ng \\
Deleted: and \\
\hline
\end{tabular}

